\title{
Efficacy and safety of switching from lopinavir/r to atazanavir/r in suppressed patients receiving a LPV/r-containing HAART: ATAZIP 96-week results
}

\author{
J Mallolas ${ }^{1}$, D Podzamczer ${ }^{2}$, P Domingo ${ }^{3}$, P Echeverría ${ }^{4}$, E Ribera ${ }^{5}$, \\ F Gutierrez ${ }^{6}$, H Knobel ${ }^{7}$, J Cosín ${ }^{8}$, E Ferrer ${ }^{2}$, JA Arranz ${ }^{9}$, V Roca10, J Pich ${ }^{11}$, \\ E de Lazzari ${ }^{11}$ and JM Gatell*1
}

\begin{abstract}
Address: ${ }^{1}$ Hospital Clinic Universitari, Barcelona, Spain, ${ }^{2}$ Hospital de Bellvitge, Barcelona, Spain, ${ }^{3}$ Hospital de Sant Pau, Barcelona, Spain, ${ }^{4}$ Fundacio IrsiCaixa, Barcelona, Spain, ${ }^{5}$ Hospital Vall d'Hebrón, Barcelona, Spain, ${ }^{6}$ Hospital General Universitario de Elche, Elche, Spain, ${ }^{7}$ Hospital del Mar, Barcelona, Spain, ${ }^{8}$ Hospital Gregorio Marañón, Madrid, Spain, ${ }^{9}$ Hospital Principe de Asturias, Madrid, Spain, ${ }^{10}$ Hospital Clínico San Carlos, Madrid, Spain and ${ }^{11}$ Hospital Clínic Universitari, Barcelona, Spain

* Corresponding author
\end{abstract}

\author{
from Ninth International Congress on Drug Therapy in HIV Infection \\ Glasgow, UK. 9-13 November 2008 \\ Published: 10 November 2008 \\ Journal of the International AIDS Society 2008, I I (SuppI I):P53 doi:I0.I I86/I758-2652-I I-SI-P53
}

This abstract is available from: http://www.jiasociety.org/content/I I/SI/P53

(c) 2008 Mallolas et al; licensee BioMed Central Ltd.

\section{Purpose of the study}

To compare the efficacy and safety of switching from lopinavir/r $(\mathrm{LPV} / \mathrm{r})$ to atazanavir/r (ATV/r) in suppressed patients vs. continuing a LPV/r HAART at 2 years.

\section{Methods}

Randomized, open-label 96-week trial including patients with virological suppression $(<200$ copies $/ \mathrm{ml} ;>=6$ months) on a LPV/r-containing HAART. Patients were randomized 1:1 to either continue LPV/r or switch to ATV/ r. Patients with $>4$ PI-associated mutations and/or failed to $>2$ PI-containing regimens were excluded. Primary endpoint: proportion of patients with treatment failure (ITT switching $=$ failure) at 48 weeks. Virologic failure: two consecutive viral load $>200$ copies/ml (OT).

\section{Summary of results}

248 patients were assigned to continue LPV/r $(n=127)$ or to switch to ATV/r $(\mathrm{n}=121)$. Baseline characteristics were balanced, including age, sex, risk for HIV infection, liver enzymes, fasting lipid profile, time on prior ARV, previous exposure to PI-containing regimens, and median CD4 (around 450 cells $/ \mathrm{mm} 3$ ). 30\% had available evidence of harboring one or more PI-associated mutations (with $10 \%$ showing at least one major mutation, IAS defini- tion). Treatment failure occurred in $30 \%(40 / 127)$ in the $\mathrm{LPV} / \mathrm{r}$ arm and in 25\% (33/121) in the ATV/r arm (difference $-4.2 \%$; $95 \%$ CI from $-15.6 \%$ to $7.1 \%)$. Virological failure occurred in $9 \%(12 / 127)$ in the LPV/r arm and in $8 \%(11 / 121)$ in the ATV/r arm (difference $-0.36 \% ; 95 \%$ CI from -7.6 to $6.9 \%$ ). Median CD4 changes from baseline were 100.5 and 40 cells/mm3 in the LPV/r and ATV/r arm, respectively. Adverse events leading to study drug discontinuation occurred in $6 \%$ in the $\mathrm{LPV} / \mathrm{r}$ arm and $5 \%$ in the ATV/r arm. Fasting TG and total cholesterol decreased significantly in the ATV/r arm, -53 and $-24 \mathrm{mg} / \mathrm{dL}$, respectively. Changes in ALT/AST were similar in both arms and significant total bilirubin elevations were frequently seen in the ATV/r arm.

\section{Conclusion}

Switching to ATV/r in virologically suppressed patients who were receiving a LPV/r-containing HAART provided comparable and durable efficacy with improved lipid parameters. 